\title{
High-energy Nd:YAG laser system with arbitrary sub-nanosecond pulse shaping capability
}

\author{
R. A. MeiJer, ${ }^{1}$ A. S. Stodolna, ${ }^{1}$ K. S. E. Eikema, ${ }^{1,2}$ and S. Witte ${ }^{1,2, *}$ \\ ${ }^{1}$ Advanced Research Center for Nanolithography (ARCNL), Science Park 110, 1098 XG Amsterdam, The Netherlands \\ ${ }^{2}$ LaserLaB, Department of Physics and Astronomy, Vrije Universiteit, De Boelelaan 1105, 1081 HV Amsterdam, The Netherlands \\ ${ }^{*}$ Corresponding author: witte@arcnl.nl
}

Received 15 May 2017; revised 17 June 2017; accepted 19 June 2017; posted 20 June 2017 (Doc. ID 295757); published 10 July 2017

We report on a laser system capable of generating highenergy (>270 mJ) temporally shaped pulses at $1064 \mathrm{~nm}$ with 0.43 -ns shaping resolution. The pulses are generated by modulation of a continuous-wave seed laser and subsequent amplification by a dual-stage grazing-incidence Nd:YVO4 "bounce" amplifier and a Nd:YAG power amplifier (all quasi-continuous diode-pumped). The system produces pulses with a high-quality top-hat spatial beam profile with up to $0.6 \mathrm{GW}$ of peak power and $44 \mathrm{~W}$ of average power, a power stability of $0.22 \% \mathrm{rms}$, and fully programmable complex temporal shapes. () 2017 Optical Society of America

OCIS codes: (140.0140) Lasers and laser optics; (140.3580) Lasers, solid-state; (140.3480) Lasers, diode-pumped; (140.3280) Laser amplifiers; (140.3538) Lasers, pulsed; (320.5540) Pulse shaping.

https://doi.org/10.1364/OL.42.002758

Laser pulses are a powerful tool for inducing a large diversity of dynamical phenomena in matter. Temporal shaping of laser pulses and pulse sequences has been used in a wide variety of fields and applications to control such dynamics. Shaping of femtosecond and picosecond pulses is generally performed in the spectral domain [1] using spatial light modulators or acousto-optic programmable dispersive filters; it is often aimed towards coherent control and photochemistry [1-3]. Shaping on the nanosecond to millisecond timescales is generally done by acousto- or electro-optic modulation and is mostly aimed towards ablation mechanics in, for example, micromachining $[4,5]$. Other fields employing temporal pulse shaping based on varying methods of pulse combining include fusion ignition [6], x-ray lasers [7], and laser-produced plasma (LPP) extremeultraviolet (EUV) sources [8].

We aim to improve control over laser-produced plasmas, and specifically their EUV emission characteristics. Such plasmas display rich dynamics on the nanosecond timescale [9], and the ability to control plasma parameters by optical means requires a combination of high-energy laser pulses with GHzbandwidth pulse shaping capabilities. Yet this intermediate regime of pulse shaping on (sub-)nanosecond timescales, especially in combination with high-energy pulses, has to the best of our knowledge remained largely unexplored. Passive shaping methods based on coherent or incoherent pulse combining have been used to generate complex pulse shapes in this parameter range. Yet these methods are inflexible, as they require precise adjustment of an array of different optical paths, and are intended to produce only one or a few specific pulse shapes $[10,11]$.

The development of fast, compact, integrated electro-optic modulators (EOM) has made arbitrary temporal shaping at $\mathrm{GHz}$ speed accessible $[12,13]$ and has proven capable of bridging the gap between spectral-domain shaping of ultrafast pulses and temporal modulation by acousto-optic modulators (AOM) and Pockels cells. However, the penalty for their fast modulation speed is a low average power threshold, usually in the milliwatt regime. Therefore, to apply temporal shaping on the 0.1-10 ns timescale to high-energy laser pulses, amplification after shaping is necessary. Several systems have already been developed, aimed at high average power $[12,14]$ and peak powers reaching tens of killowatts.

In this Letter, we present a laser system delivering arbitrary shaped pulses with 0.43 -ns resolution and pulse energies ranging between 270 and $440 \mathrm{~mJ}$ at 1064-nm wavelength and $100-\mathrm{Hz}$ repetition rate. The highest achieved peak power is $0.6 \mathrm{GW}$, at an average power of $27 \mathrm{~W}$ and pulse duration of $0.43 \mathrm{~ns}$. The system is based on a modulated continuous-wave (CW) laser which seeds a dual-stage grazing-incidence amplifier utilizing $\mathrm{Nd}: \mathrm{YVO}_{4}$ crystals pumped at $880 \mathrm{~nm}$ [15]. Further amplification is obtained by using a quasi-continuous-wave (QCW) diode-pumped Nd:YAG power amplifier. The laser output is characterized, and several examples of pulse shapes are presented showing the complex pulse shaping capabilities.

Figure 1 shows a schematic of the full laser system in which the pulse shaping, pre-amplifier, and power amplification stages are indicated by their respective frames. The pulse shaping section is fully fiber coupled (polarization maintaining) and starts with a CW fiber laser (NKT Koheras BoostiK Y10) delivering $2 \mathrm{~W}$ of continuous laser light at $1064 \mathrm{~nm}$. This light is first gated by an AOM (Gooch \& Housego Fiber-Q, operating at a $150-\mathrm{MHz}$ carrier frequency), resulting in square pulses. The function of the AOM is to deliver high-contrast modulation $(50 \mathrm{~dB})$, minimizing parasitic seed light, and simultaneously limit the average input power to two high-speed EOMs following it. 


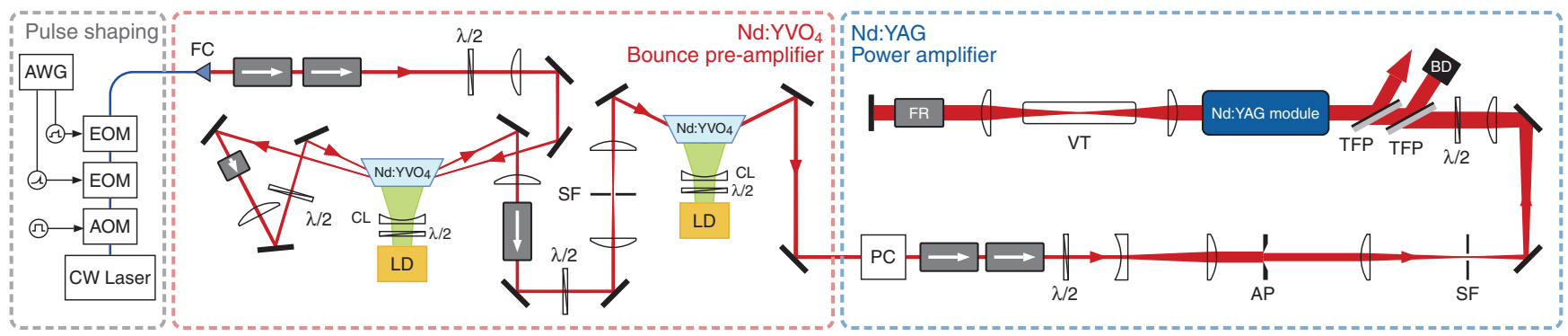

Fig. 1. Schematic of the all-fiber pulse shaping chain, the dual-stage $\mathrm{Nd}: \mathrm{YVO}_{4}$ bounce pre-amplifier, and Nd:YAG power amplifier (AOM, acousto-optic modulator; EOM, electro-optic modulator; AWG, arbitrary waveform generator; FC, fiber collimator; arrow, isolator; $\lambda / 2$, half-wave plate; CL, cylindrical lens; LD, laser diode; SF, spatial filter; PC, Pockels cell; AP, aperture; TFP, thin-film polarizer; VT, vacuum tube; FR, Faraday rotator; $\mathrm{BD}$, beam dump).

Each EOM (Jenoptik AM 1064, amplitude modulator, 200-ps rise time, $30-\mathrm{dB}$ dynamic range) is individually driven by one of the two channels of a 2.3-Gs/s arbitrary waveform generator (AWG) (Tabor Electronics WX2182C). Working in tandem, they create the final pulse shape to be amplified. A second EOM was necessary to improve contrast by effectively blocking light leaking through the first EOM and to improve pulse shaping at the rising edge of a pulse, where the gain saturation effect present in the amplifiers is the strongest.

The shaped pulses are initially amplified by a dual-stage triple-pass grazing-incidence "bounce" pre-amplifier, containing two $\mathrm{Nd}: \mathrm{YVO}_{4}$ crystals (a-cut) with doping percentages of $1 \%$ and $0.5 \%$ and dimensions of $5 \mathrm{~mm} \times 2 \mathrm{~mm} \times 20 \mathrm{~mm}$ and $6 \mathrm{~mm} \times 4 \mathrm{~mm} \times 20 \mathrm{~mm}$, respectively. The crystals are optically pumped from the side by QCW diode arrays (Dilas MY-Series), thermally tuned to around $50^{\circ} \mathrm{C}$ to emit at the upper lasing level of $\mathrm{Nd}: \mathrm{YVO}_{4}$ around $880 \mathrm{~nm}$, delivering approximately $240 \mathrm{~W}$

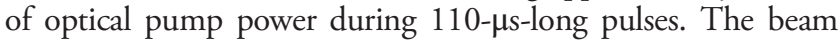
enters the Nd:YVO 4 crystals through the side facet and undergoes total internal reflection on the pumped surface of the crystal, the "bounce." This geometry allows for strong pumping and provides homogeneous amplification of the seed, preventing spatial distortion of the beam profile. Note that the side facets of the crystals have a small angle of $5^{\circ}$ to prevent parasitic lasing in the crystal. Furthermore, optical isolators and a spatial filter are placed between the passes to block back-reflections, keeping amplified spontaneous emission (ASE) to a minimum, and preventing parasitic self-lasing of the amplifier. The available seed energy depends on chosen pulse duration and (pre-compensated) pulse shape. Given the 2-W output from the CW laser and assuming a specified insertion loss of the three modulators together of $12 \mathrm{~dB}$, an upper limit for the energy of seed pulses of known shape can be determined. For example, a 500-ns square seed pulse leads to an estimated seed energy of $63 \mathrm{~nJ}$, which is amplified to $2.84 \mathrm{~mJ}$ by the total of three amplification passes. This corresponds to a saturated gain of $46.5 \mathrm{~dB}$ for the full bounce amplifier. A 0.5 -ns full width at half-maximum (FWHM) Gaussian pulse is estimated to have an energy of $30 \mathrm{pJ}$ and is amplified to $0.4 \mathrm{~mJ}$ at a gain of $71 \mathrm{~dB}$.

After the bounce amplifier, the beam is further amplified in a power amplification stage, which is separated from the pre-amplifier by a Pockels cell acting as a temporal gate to suppress ASE. In this power amplifier we make use of a QCW diode-pumped Nd:YAG module (Northrop Grumman REA6308) containing a $6.35-\mathrm{mm}$ diameter Nd:YAG rod $146 \mathrm{~mm}$ in length. Nd:YAG is used here as a gain medium instead of $\mathrm{Nd}: \mathrm{YVO}_{4}$ because of its higher energy storage capability, albeit providing less gain than $\mathrm{Nd} \mathrm{YVO}_{4}[16]$. Before passing through the module, the Gaussian beam is expanded slightly to a $1.8-\mathrm{mm}$ FWHM and clipped by a 2.4-mm hard aperture. This controlled clipping of the beam prevents further unwanted clipping of the beam at the edges of the Nd:YAG rod and allows for a better control of the filling fraction of the rod. An initial relay telescope ( $f=200 \mathrm{~mm}$ and $f=400 \mathrm{~mm}$ ), with a weak spatial filter $(2.5-\mathrm{mm}$ diameter) at the focus, images the aperture plane near the end face of the module with 2 times magnification, leading to a 4.8-mm beam size in the module. Increasing the filling fraction of the Nd:YAG rod will evidently result in a higher output energy, but also to beam distortion due to the fivefold side pumping geometry utilized in the module that gives an inhomogeneous gain profile near the outer edge. A 1:1 relay telescope, with a vacuum tube at the focus to prevent breakdown in air, images the previous image plane onto the end mirror, which reflects the beam for the second pass. Between the two passes through the module, the polarization is rotated by a Faraday rotator. In combination with relay imaging, this neutralizes the effects of thermal birefringence in the Nd:YAG crystal rod [17]. A thin-film polarizer (TFP) is used to couple out the beam, while an additional TFP has been inserted to increase isolation and prevent parasitic lasing. The temperature of the Nd:YAG rod influences its pump absorption, which provides some control over the radial gain profile in the rod. This profile is such that the gain increases towards the edges of the rod. By seeding with a clipped Gaussian input shape and controlling both the rod surface temperature and pump power, we can optimize the output beam profile to obtain a high-quality flat top, as can be seen in Fig. 2(a). We drive the module with $250-\mu$ s pulses at $110 \mathrm{~A}$ and operate the system at a cooling temperature of $20^{\circ} \mathrm{C}$. Depending on input pulse shape and energy we achieve a gain between 23.9 and $30.6 \mathrm{~dB}$, corresponding to output pulse energies between 270 and $440 \mathrm{~mJ}$. The limiting factor in the achievable gain of the present doublepass amplifier configuration is the occurrence of self-lasing at higher pump currents. A route towards further power scaling can be the implementation of an additional double-pass amplifier stage with increased rod diameter. Since the gain bandwidths of Nd: $\mathrm{YVO}_{4}$ and Nd:YAG are not identical, we adjust the wavelength of the CW source at sub-nanometer scale such that the overall output energy is optimized.

Figure 2(b) shows the measured output stability of the complete all-diode-pumped system. A power stability of $0.22 \% \mathrm{rms}$ 
(a)
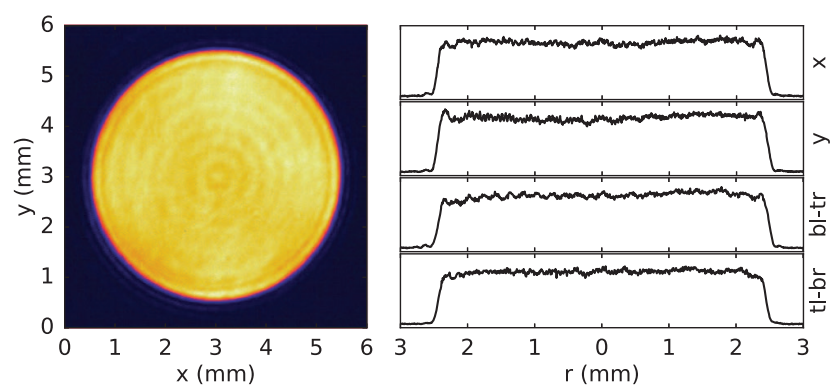

(b)

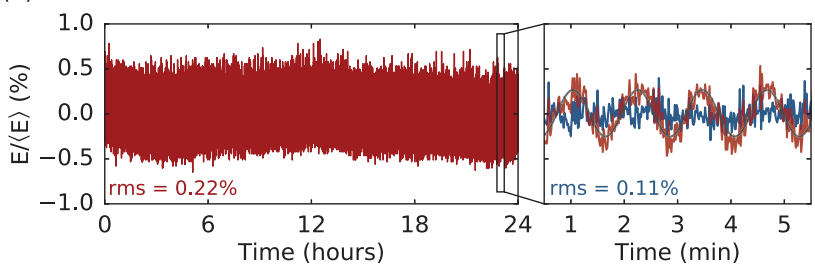

Fig. 2. (a) Near-field beam profile of the amplified pulses measured with $1: 1$ relay imaging at the output of the system. Horizontal, vertical, and diagonal cross sections (from bottom left to top right and top left to bottom right) are included. Diffraction rings caused by spatial filtering before amplification in the power amplifier are visible, as well as a weak cross geometry due to remaining thermally induced birefringence in the Nd:YAG rod. Small-scale diagonal fringes present in the image are an artifact from interference in the charge-coupled device (CCD) camera (they are mostly visible in the cross sections as small-scale ripples). (b) Long $(24 \mathrm{~h})$ and short $(5 \mathrm{~min})$ time duration stability of the output pulse energy.

over $24 \mathrm{~h}$ was obtained for the pulse shown in 3(a) with $437 \mathrm{~mJ}$ output energy. The long-term stability is limited to $0.22 \% \mathrm{rms}$ by the cooling cycle $(\approx 1.2 \mathrm{~min})$ of the $\mathrm{Nd}$ :YAG module in the power amplifier, as can be seen from the zoom-in. By subtracting this oscillation we find a short-term power stability of $0.11 \% \mathrm{rms}$

The advanced temporal shaping capabilities of our laser system are demonstrated in Fig. 3. Figure 3(a) shows the measured pulse shape produced by the amplifiers for a square 500-ns input pulse, showing gain depletion from the amplifiers within only a few nanoseconds. As a result, the output pulse is asymmetric with a duration reduced to only 1.2 ns FWHM. With active shaping, we achieve a minimum pulse duration of 0.43 ns FWHM, as shown in Fig. 3(b). Figures 3(c)-3(h) display several pulse shapes over a time span of $10 \mathrm{~ns}$, demonstrating the fast pulse shaping capabilities. More specifically, Fig. 3(c) shows a pulse train demonstrating the shaping resolution obtained by alternating consecutive sample points in the AWG waveform. The average distance between the peaks and valleys is found to be $0.43 \mathrm{~ns}$. This, as well as the minimum pulse length of $0.43 \mathrm{~ns}$, corresponds very accurately to the sample rate of the AWG of 2.3 GHz. Furthermore, as shown in Fig. 3(j), the system can generate pulses up to $1 \mu$ s (limited by the opening time of the Pockels cell), spanning 4 orders of magnitude in pulse length. The temporal intensity of the $1-\mu$ s pulse remains very constant; it deviates from a perfect flat intensity profile by just $2.9 \%$ rms over the full pulse duration. For shorter square pulses, such as the 20-ns pulse shown in Fig. 3(i), we achieved deviations from a perfect flat temporal profile of only $1.6 \% \mathrm{rms}$. To illustrate the level of suppression necessary to compensate for
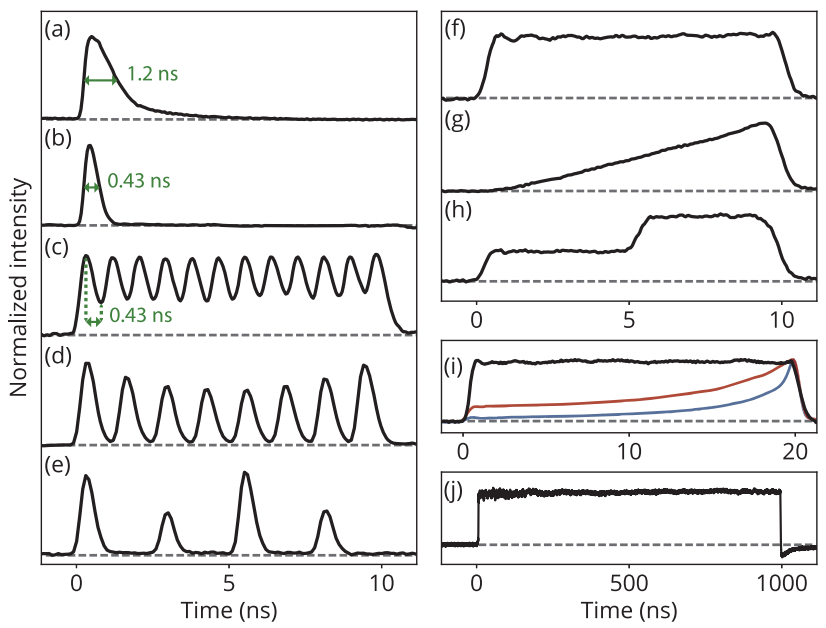

Fig. 3. Measured temporal pulse shapes demonstrating the shaping capabilities of the laser system. Including (a) the response of the system when given a flat-intensity input pulse, (b) the shortest pulse obtained using active shaping with a FWHM of $0.43 \mathrm{~ns}$, (c)-(e) varying pulse trains, (f) a square 10-ns pulse, (g) a linear rising slope, (h) a 2-step flat pulse, (i) a square 20 -ns pulse, and (j) a $1-\mu$ s square pulse. In (i) the blue and red curves are the waveforms applied to the first and second EOM, respectively. The dip at the end of the $1-\mu$ s pulse is attributed to the photodiode response. All pulses are measured using a $>15-\mathrm{GHz}$ photodiode positioned at a focus and a $4-\mathrm{GHz}$ oscilloscope. All traces shown are averaged over 16 pulses.

temporal distortion, we have added the corresponding waveforms applied to the EOMs for this case.

In the present configuration, the achievable output energy from the amplifier system exhibits a significant dependence on the target pulse shape. An example is given in Fig. 4, which shows the measured pulse energy for pulses with a square intensity profile of different duration. A maximum output energy of $400 \mathrm{~mJ}$ is found at a pulse duration of $5 \mathrm{~ns}$. The observed energy increase for short pulses is readily explained by the increasing seed energy that is available when the pulse becomes longer. To understand the decrease for pulse durations above 5 ns, we modeled the gain dynamics in a double-pass amplifier [18] for the case of square output pulses of different durations. The input pulses were

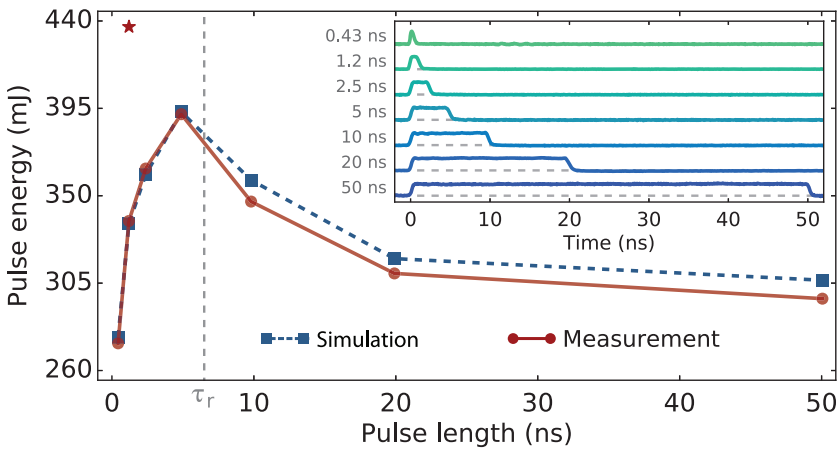

Fig. 4. Measured and simulated pulse energy as a function of pulse length for output pulses with a square intensity profile. The traces of the corresponding measured pulses are shown in the inset. The starmarked data point corresponds to the saturated response of the system as shown in Fig. 3(a). For pulse lengths above 50 ns, the curve flattens out at approximately $300 \mathrm{~mJ}$. 
approximated by an exponential shape (increasing in time) with energies equal to the measured values before the first pass of the $\mathrm{Nd}$ :YAG module. Although the assumed single exponential input pulse shape is not fully accurate, the results explain the trend in energy accurately. The time at which the drop in energy occurs corresponds to the round-trip time $\tau_{r}$ of a light pulse through the power amplifier, in our case approximately 6.5 ns. For pulse durations longer than $6.5 \mathrm{~ns}$, the leading edge of the pulse will have traversed the Nd:YAG rod twice before the trailing edge has completed the first pass. This effect causes gain stripping for parts of the pulse $>6.5 \mathrm{~ns}$, and leads to a lower overall gain and reduced energy extraction efficiency for longer pulses. The data points for pulse lengths $<\tau_{r}$ (where pulse shape has no influence) were fitted to obtain an effective saturation fluence for this amplification stage of $0.83 \mathrm{~J} / \mathrm{cm}^{2}$. With a higher available seed intensity, the effects of gain stripping due to the described pulse overlap can be averted by fully saturating the power amplifier. At the current repetition rate of $100 \mathrm{~Hz}$, and in combination with the AOM as a first temporal gating device, the average input power to the EOMs is still several orders of magnitude below their specified damage threshold. Therefore, there is still room to increase the available seed pulse energy to the amplifiers by using a more intense seed laser, such as a pulsed diode source [14]. The round-trip time of the double pass through the first crystal of the bounce amplifier is approximately 3 ns. However, any significant drop in output energy from the bounce amplifier due to pulse overlap was absent. This is as expected, as there is no significant energy extraction in this amplification stage.

The strong gain dynamics require significant pre-shaping of the input pulse shape to obtain a desired output shape. In a first implementation these shapes have been manually programmed and optimized. As a first step towards automated optimization of a target output shape, we have implemented a feedback algorithm that optimizes towards basic pulse shapes. This algorithm uses a straightforward approach, optimizing temporal sections of the pulse from early to later times while setting the intensity of each section relative to preceding sections, under the assumption that each point in the intensity profile only influences later parts of the pulse. Although, as already discussed above, this assumption is not fully justified in a double-pass amplifier, the approach works well when sufficient dynamic range is used and gain depletion is avoided before the end of the pulse is reached. In addition to setting specific pulse shapes, we envision the use of evolutionary algorithms to enable automatic optimization to any complex shape, using specific experimental parameters to define a fitness function $[5,19]$.

The ability to temporally tailor nanosecond pulses with the demonstrated temporal resolution and range makes this a versatile system for high-energy laser-matter interaction. A possible application that we envision involves temporal control over the vaporization of a metal target by an optimized laser pulse shape. The target can be heated up in a controlled way by adjusting the temporal pulse shape and pulse energy. In these conditions, the metal target adiabatically follows an equilibrium vaporization path through the phase diagram and avoids both super-heating and subsequent phase explosion, which are commonly observed for high-energy laser-matter interaction [20-22]. This approach could lead to a slowly expanding gas target with a homogeneous density profile without significant generation of ions or nanoparticles, which would be a desirable starting point for laser-produced plasma experiments.
In summary, we have shown a combination of true arbitrary temporal intensity shaping of laser pulses with a resolution of $0.43 \mathrm{~ns}$, pulse energies up to $437 \mathrm{~mJ}$, an average power of $44 \mathrm{~W}$, and peak powers up to $0.6 \mathrm{GW}$. The large dynamic range in shaping achieved by two EOMs ensures that we can always achieve a combination of accurate pulse shape and good energy extraction that reaches $30 \%-40 \%$ of the stored energy. We achieve excellent beam quality and output stability independent of the produced pulse shape. Implementation of faster EOMs and driving electronics can further improve the shaping resolution. The addition of further amplifier stages, and employing a larger diameter Nd:YAG rod and higher stored energy, will allow extension of this technology to pulse energies above the Joule level, and multi-gigawatt peak powers.

Funding. This work has been carried out at the Advanced Research Center for Nanolithography (ARCNL); a publicprivate partnership of the Universiteit van Amsterdam (UvA); the Vrije Universiteit Amsterdam (VU); the Nederlandse Organisatie voor Wetenschappelijk Onderzoek (NWO); and the Semiconductor Equipment Manufacturer ASML.

\section{REFERENCES}

1. D. Goswami, Phys. Rep. 374, 385 (2003).

2. W. S. Warren, H. Rabitz, and M. Dahleh, Science 259, 1581 (1993).

3. A. Assion, Science 282, 919 (1998).

4. K. Pangovski, M. Sparkes, A. Cockburn, W. O'Neill, P. S. Teh, D. Lin, and D. Richardson, IEEE J. Sel. Top. Quantum Electron. 20, 51 (2014).

5. P. Deladurantaye, D. Gay, A. Cournoyer, V. Roy, B. Labranche, M. Levesque, and Y. Taillon, Proc. SPIE 7195, 71951S (2009).

6. O. A. Hurricane, D. A. Callahan, D. T. Casey, P. M. Celliers, C. Cerjan, E. L. Dewald, T. R. Dittrich, T. Döppner, D. E. Hinkel, L. F. B. Hopkins, J. L. Kline, S. Le Pape, T. Ma, A. G. MacPhee, J. L. Milovich, A. Pak, H.-S. Park, P. K. Patel, B. A. Remington, J. D. Salmonson, P. T. Springer, and R. Tommasini, Nature 506, 343 (2014).

7. G. V. Cojocaru, R. G. Ungureanu, R. A. Banici, D. Ursescu, O. Guilbaud, O. Delmas, A. Le Marec, O. Neveu, J. Demailly, M. Pittman, S. Kazamias, S. Daboussi, K. Cassou, L. Li, A. Klisnick, P. Zeitoun, and D. Ros, Opt. Express 24, 14260 (2016).

8. K. M. Nowak, Y. Kurosawa, T. Suganuma, Y. Kawasuji, H. Nakarai, T. Saito, J. Fujimoto, and H. Mizoguchi, Opt. Lett. 41, 3118 (2016).

9. S. Yuspeh, K. L. Sequoia, Y. Tao, M. S. Tillack, R. A. Burdt, and F. Najmabadi, Appl. Phys. Lett. 96, 261501 (2010).

10. J. Fülöp, Z. Major, B. Horváth, F. Tavella, A. Baltuška, and F. Krausz, Appl. Phys. B 87, 79 (2007).

11. Y. Kong, Q. Z. Liu, C. Deng, X. J. Huang, and G. H. Chen, J. Lightwave Technol. 27, 3017 (2009).

12. A. Malinowski, K. T. Vu, K. K. Chen, J. Nilsson, Y. Jeong, S. Alam, D. Lin, and D. J. Richardson, Opt. Express 17, 20927 (2009).

13. C. E. Rogers and P. L. Gould, Opt. Express 24, 2596 (2016).

14. M. Nie, Q. Liu, E. Ji, X. Cao, X. Fu, and M. Gong, Opt. Lett. 42, 1051 (2017).

15. J. Morgenweg and K. S. E. Eikema, Laser Phys. Lett. 9, 781 (2012).

16. W. Koechner, Solid-State Laser Engineering, 6th ed. (Springer, 2014).

17. J. Sherman, Appl. Opt. 37, 7789 (1998).

18. J. Jeong, S. Cho, and T. J. Yu, Opt. Express 25, 3946 (2017).

19. T. Baumert, T. Brixner, V. Seyfried, M. Strehle, and G. Gerber, Appl. Phys. B 65, 779 (1997).

20. A. Pakhomov, M. Thompson, and D. Gregory, J. Phys. D 36, 2067 (2003).

21. Y. Tao, M. S. Tillack, S. S. Harilal, K. L. Sequoia, and F. Najmabadi, J. Appl. Phys. 101, 023305 (2007).

22. Y. Tao, Y. Ueno, S. Yuspeh, R. Burdt, M. S. Tillack, and F. Najmabadi, Proc. SPIE 7969, 796930 (2011). 